\title{
Wannier-Stark resonances in semiconductor superlattices
}

\author{
M. Glück, A. R. Kolovsky[*], H. J. Korsch, and F. Zimmer \\ Fachbereich Physik, Universität Kaiserslautern, D-67653 Kaiserslautern, Germany
}

(Dated: June 6, 2001)

\begin{abstract}
Wannier-Stark states for semiconductor superlattices in strong static fields, where the interband Landau-Zener tunneling cannot be neglected, are rigorously calculated. The lifetime of these metastable states was found to show multiscale oscillations as a function of the static field, which is explained by an interaction with above-barrier resonances. An equation, expressing the absorption spectrum of semiconductor superlattices in terms of the resonance Wannier-Stark states is obtained and used to calculate the absorption spectrum in the region of high static fields.
\end{abstract}

\section{INTRODUCTION}

Semiconductor superlattices (SL) are the traditional object for studying Wannier-Stark states (WS-states) which, by definition, are the metastable states of a charged particle in a periodic potential plus static electric field [1]

$$
H=p^{2} / 2 m+V(x)+e F x, \quad V(x+d)=V(x) .
$$

The first observation of the Wannier-Stark spectrum in a semiconductor superlattice in 1988 [2] ended a long theoretical discussion about the physical relevance of WS-states. Now it is commonly accepted that they are the resonances states of the system. However, in theoretical analyses of related problems their metastable character is most often neglected and they are approximated by stationary states (single-band, tight-binding, and similar approximations). For a weak static field this approximation can be justified because the tunneling lifetime of WS-states essentially exceeds other characteristic times of the system, in particular, the relaxation time due to scattering of the carriers by impurities. However, this is not true for strong fields, where the lifetime of WS-states can be considerably shorter than the relaxation time. Recently this regime has attracted much attention both theoretically $[3,4]$ and experimentally $[5-7]$. In particular, it was demonstrated in Ref. [4] that at high (but realistic) field strengths the concept of localized WS-states may completely fail.

In the present paper we study WS-states in SL beyond the finite-band approximation. As an analytical approach behind the numerical results presented below we use a specific scattering theory developed earlier in relation to optical superlattices [8-11]. Using this approach one finds Wannier-Stark resonances (WS-resonances) as the complex-energy poles $\mathcal{E}=E-i \Gamma / 2$ of the rigorously constructed scattering matrix. The nonhermitian eigenstates of the Hamiltonian (1) corresponding to these discrete complex energies are the metastable WS-states with the lifetime $\tau=\hbar / \Gamma$. In the limit $F \rightarrow 0$ the lifetime $\tau$ goes to infinity and the resonance WS-states approach stationary states predicted by the single-band model. Here, however, we are mainly interested in the opposite limit of a strong field, where the tunneling effects course an essential deviation from the single-band (or finite-band) approximation. It should be mentioned that the notion of strong field has a relative meaning and depends on the superlattice parameters. For example, for shallow $G a A s / A l_{x} G a_{1-x} A s$ superlattices with $d \sim 100 \AA$ and $x \sim 0.1$ the region of strong static field corresponds to $F>10$ $\mathrm{kV} / \mathrm{cm}$.

The structure of the paper is as follows. In Sec. 2 we briefly recall the basics of the scattering matrix approach used to calculate WS-resonances. Sections 3 discusses the general structure of the WS-resonances in the SL potential. It is shown that the SL potential supports many Wannier-Stark ladders (WSL) of resonances which can be classified either as under-barrier or above-barrier WSL. The interaction of different WSLs and its effect on the lifetime of WS-states is studied in Sec. 4. Sections 5 and 6 analyze the density of states and the wave functions of an electron (hole) in the SL potential. In Sec. 7 we derive an analytical expression for absorption spectrum of an undoped SL in terms of resonance WS-states and calculate the Wannier-Stark fan diagram for the semiconductor superlattices with the parameters indicated above. This section is followed by the conclusion summarizing the obtained results.

\section{SCATTERING MATRIX APPROACH}

In this section we briefly describe the approach of Ref. [11] used to find the quantum resonances in the system (1). This approach inverts the traditional solid-state-physics approach, where one treats the static field $F$ as a perturbation 
to the field-free Hamiltonian, and considers the periodic potential $V(x)$ to be a perturbation to the Stark Hamiltonian $H_{0}=p^{2} / 2 m+e F x$. In this way the notion of the scattering state $\Psi_{E}(x)$ appears which, in the limit $x \rightarrow-\infty$ (here and below we assume $F>0$ ), have an asymptotic similar to the asymptotic of the Airy function. Namely, in the momentum representation, $\Psi_{E}(k)=\int \mathrm{d} x \exp (i k x) \Psi_{E}(x)$, the asymptotic is

$$
\lim _{k \rightarrow \pm \infty} \Psi_{E}(k)=\exp \left[i\left(\frac{\hbar^{2} k^{3}}{6 m e F}-\frac{E k}{e F} \pm \theta(E)\right)\right]
$$

where the phase shift $\theta(E)$ takes into account the effect of the periodic potential. [Note, that the scattering states $\Psi_{E}(x)$ continuously depend on the energy and are normalized as $\int \Psi_{E}^{*}(x) \Psi_{E^{\prime}}(x) \mathrm{d} x=\delta\left(E-E^{\prime}\right)$.] The phase shift $\theta(E)$ in Eq. (2) defines the scattering matrix of the system, $S(E)=\exp [-i 2 \theta(E)]$. By definition, the quantum resonances are the poles of the analytical continuation of the scattering matrix into the complex plane [12]. It has been proven that for the Wannier-Stark Hamiltonian (1) the resonances are arranged in the ladder

$$
\mathcal{E}_{\alpha, l}=E_{\alpha}+e d F l-i \Gamma_{\alpha} / 2
$$

In Eq. (3) $l=0, \pm 1, \ldots$ is the site index, associated with $l$-th well of the periodic potential, and $\alpha=1,2, \ldots$ is the Bloch-band index. It can be also shown that in the limit $F \rightarrow 0$ the following relations hold:

$$
E_{\alpha} \approx \bar{E}_{\alpha} \equiv \int E_{\alpha}(\kappa) \mathrm{d} \kappa
$$

where $E_{\alpha}(\kappa)$ stands for the dispersion relation of the $\alpha$-th Bloch band at $F=0$; and

$$
\Gamma_{\alpha} \propto F \exp \left(-C_{\alpha} / F\right)
$$

where the constant $C_{\alpha}$ is defined by the energy gap between $\alpha$ and $\beta=\alpha+1$ Bloch bands [13]. We would like to stress that the displayed relations are estimates. Due to the ladder interaction (see Sec. III) the actual dependence $\Gamma_{\alpha}=\Gamma_{\alpha}(F)$ has oscillations superimposed on the smooth dependence (5). Simultaneously, there are deviation from the single-band result (4) and the resonance position $E_{\alpha}$ is a function of $F$. In paper [8] we suggested a simple numerical method (justified later on in Ref. [11]) which, in principle, allows to find WS-resonances for an arbitrary periodic potential $V(x)$. This method maps the eigenvalue problem for the Hamiltonian $H$ to the eigenvalue problem for the evolution operator $U\left(T_{B}\right)=\exp \left(-i H T_{B} / \hbar\right)$ over the Bloch period $T_{B}=2 \pi \hbar / e d F$ [14]. In other words, one starts from the eigenvalue equation

$$
U\left(T_{B}\right) \Phi_{E}(x)=\lambda \Phi_{E}(x), \quad \lambda=\exp \left(-i \frac{2 \pi E}{e d F}\right) .
$$

Then the poles of the scattering matrix are found as the complex eigenvalues of the matrix of the evolution operator calculated in the basis of the periodic functions $\langle x \mid n\rangle=(2 \pi)^{-1 / 2} \exp ($ inx $)$ and truncated to a finite size $|n|<N \rightarrow \infty$. (In practice, it is enough to keep the numerical parameter $N$ large enough.) Note, that the energy $E$ in Eq. (6) is defined up to multiples of $\Delta E=e d F$. Thus the whole ladder of resonances (3) corresponds to a single eigenvalue $\lambda$ of the evolution operator matrix.

A few words should be added about the metastable WS-states. As mentioned in Sec. 1, these states satisfy the equation

$$
H \Psi_{\alpha, l}(x)=\mathcal{E}_{\alpha, l} \Psi_{\alpha, l}(x)
$$

where $\mathcal{E}_{\alpha, l}$ are the complex energies in Eq. (3). Because WS-states are nonhermitian states of the Hamiltonian (1), one should distinguish the left $\Psi_{\alpha, l}^{L}(x)$ and the right $\Psi_{\alpha, l}^{R}(x)$ eigenfunctions. Formally they correspond to scattering states with zero amplitude of incoming or outgoing waves and diverge exponentially for $x \rightarrow-\infty(F>0)[12]$. Nevertheless, the scalar product of the left and right eigenfunctions is finite. In the results presented below we normalize the resonance wave functions as

$$
\int \Psi_{\alpha, l}^{R}(x) \Psi_{\beta, m}^{L}(x) \mathrm{d} x=\delta_{\alpha, \beta} \delta_{l, m}
$$




\section{UNDER- AND ABOVE-BARRIER WS-RESONANCES}

We proceed with the analysis of WS-resonances in the SL potential. In the simplest (traditional) approach the SL potential is modeled by the periodic square-shaped function

$$
V(x)=\left\{\begin{array}{ll}
V & , \quad|x| \leq a / 2 \\
0 & , a / 2<|x| \leq d / 2
\end{array},\right.
$$

where $a$ and $b=d-a$ are the thickness of alternating semiconductor layers. To be concrete, we shall analyze the electron WS-resonances and choose the following SL parameters: $d=114 \AA, a=b, V^{e}=0.0632 \mathrm{eV}, m^{e}=0.067$ of the electron mass and the effective electron mass is assumed to be independent of the coordinate $x$. For the purpose of future use the Bloch band spectrum of the system is depicted in Fig. 1(b).

Figure 2(b) shows the widths $\Gamma_{\alpha}$ of WS-resonances belonging to the seven most stable WSLs as a function of $1 / F$. It is seen in the figure that for a low static field $F \lesssim 10 \mathrm{kV} / \mathrm{cm}$ the lifetime of the ground WSL essentially exceeds the lifetime of the next six WSLs, which form a wide irregular band. However, for a higher field $F \gtrsim 10$ $\mathrm{kV} / \mathrm{cm}$ the resonances belonging to different WSLs have comparable widths and, therefore, the notion of the ground WSL loses its absolute meaning. The left panel in Fig. 2 shows the relative energy $E_{1}$ of the most stable WSL, reduced to the fundamental energy interval $-e d F / 2 \leq E<e d F / 2$. The slope of the straight line approximating the displayed dependence is given by the absolute energy $E_{1}^{*}$ of the ground WS-resonances in the limit $F \longrightarrow 0$. For the chosen superlattice parameter it is $E_{1}^{*}=0.039 \mathrm{eV}$, which should be compared with the height of the potential barrier $V^{e}=0.0632 \mathrm{eV}$ and "mean position" of the ground Bloch band $\bar{E}_{1}=0.0383 \mathrm{eV}$. Because $E_{1}^{*} \approx \bar{E}_{1}<V^{e}$ these resonances can be identified (at least for a low static fields) as an under-barrier resonances originating from the ground Bloch band.

It is also seen in Fig. 2(a) that for a low field the dependence $E_{1}=E_{1}(F)$ is a continuous function of $F$ but it has a number of discontinuities in the region of high static field. This is explained by the phenomenon of avoided level crossing in the complex plane. In fact, for the complex eigenvalues depending on some control parameter two kinds of such crossings are possible - either the real parts of the eigenvalues cross and the imaginary parts show an avoided crossing or, vice versa, the imaginary parts cross but the real parts show an avoided crossing (see [15], for example). The depicted result indicate that for a low field, where $\Gamma_{1} \ll \Gamma_{\alpha}(\alpha>1)$, true crossings of the level $E_{1}$ with other levels occur. Opposite to this, for a high field, the anticrossings mainly take place. We shall come back to this problem in Sec. IV.

Perhaps the most remarkable feature of the analyzed data is the strong oscillation of the resonance width of the ground WS-resonances. These oscillations are superimposed on the general tendency (5) and show an hierarchical structure of different scales. As shown below, the physical origin of the resonance width oscillations is attributed to the large number of crossings with WSLs associated with the unstable above-barrier resonances.

To understand the structure of the above-barrier resonances in semiconductor superlattices it is convenient to consider the square-box potential (9) as the limit $M \rightarrow \infty$ of the finite Fourier series

$$
V_{M}(x)=V^{e}\left[\frac{1}{2}+\sum_{m=1}^{M} v_{m} \cos \left(\frac{2 \pi m}{d} x\right)\right] \quad, v_{m}=\frac{2}{\pi m} \sin \left(\frac{\pi m}{2}\right),
$$

(here we consider the case $a=b=d / 2$.) Then the structure of the above-barrier resonances can be qualitatively described in terms of the Landau-Zener theory, where the lifetime of WS-states is determined by the probability of tunneling between the adjacent bands.

The first row in Fig. 3 shows the relative energies and widths of the ground under-barrier and first above-barrier resonances for $M=1$, i.e. a pure cosine-potential, and $0.1<1 / F<0.15 \mathrm{~cm} / \mathrm{kV}$. The absolute energy of the resonances, obtained from the slopes of the lines, are $E_{1}^{*}=0.039 \mathrm{eV}$ and $E_{2}^{*}=0.134 \mathrm{eV}$, which approximately coincide with the mean position of the first $\left(\bar{E}_{1}=0.0386 \mathrm{eV}\right)$ and second $\left(\bar{E}_{2}=0.1370 \mathrm{eV}\right)$ Bloch bands [see Fig. 1(a)]. The mean slopes of the dependences for the resonance width $\Gamma_{1}$ and $\Gamma_{2}$ are given by the values of the energy gaps $\Delta_{1,2}=0.0043$ and $\Delta_{2,3}=1.58 \cdot 10^{-6} \mathrm{eV}$ at the edge and in the center of the Brillouin zone, respectively. Because the energy gaps between the other bands are exponentially small, the next above-barrier resonances have very large widths (small lifetimes) and are of a minor physical importance. It is also seen in the figure that the oscillations of the width $\Gamma_{1}$ are due to the crossings of the ground WSL with the ladder originating from the second Bloch band. Namely, each maximum of $\Gamma_{1}$ corresponds to a particular crossing of the level $E_{1}$ with the level $E_{2}$.

The second row in Fig. 3 corresponds to $M=3$. Now we have two above-barrier resonances and they are actually more stable than the single above-barrier resonance for $M=1$. This is explained by the fact that the term $v_{3} \cos (6 \pi x / d)$ in Eq. (10) opens the gap $\Delta_{3,4}=0.0132$ between the 3 -rd and 4 -th Bloch bands at the edges of the Brillouin zone [16]. [The Bloch dispersion relation for $M=3$ practically coincides for $E<1 \mathrm{eV}$ with the one shown 
in Fig. 1(b) and with the one shown in Fig. 1(a) for $E>1 \mathrm{eV}$.] Note that this gap is larger than the gap $\Delta_{2,3}$ at the center of the Brillouin zone. Because of this, the WSLs originating from the second and third band strongly interact and the corresponding above-barrier resonances appear as a "degenerate" pair with the absolute energy $E_{2}^{*}=E_{3}^{*} \approx\left(\bar{E}_{2}+\bar{E}_{3}\right) / 2$ (see next section). Similar to the case $M=1$, the crossings of the above-barrier WSLs with the ground WSL results in variation of $\Gamma_{1}$. However, since we have two more-or-less stable above-barrier resonances, the characteristic period of the ground resonance width oscillation is two times smaller.

The next nonvanishing term $v_{5} \cos (10 \pi x / d)$ in the series (10) opens the gap between the 5 -th and 6-th Bloch bands and a new pair of almost degenerate above-barrier resonances, $E_{4}^{*}=E_{5}^{*}$, appears (third row in Fig. 3 ). The presence of these "new" above-barrier resonance affect the width of the ground resonance in similar way, - each crossing of the level $E_{1}$ with levels $E_{4}$ and $E_{5}$ brings additional peak to the dependence $\Gamma_{1}(F)$.

The described process of the formation of above-barrier resonances can be continued towards $M \rightarrow \infty$ which will result in fractal-like dependence of $\Gamma_{1}(F)$. We would like to stress, however, that this fractal-like behavior is a consequence of the non-analyticity of the square-box potential reflected in the slow $1 / M$-convergence of the Fourier series. For an analytic potential, which is obviously the case realized in practice, the Fourier series (10) has an effective cut-off. Because of this, the number of physically important above-barrier WSLs is finite and there is some minimum scale of the oscillations in $\Gamma_{1}(F)$.

\section{INTERACTION OF RESONANCES}

As shown in the previous section, WS-resonances in SL may strongly affect each other [17]. Here we study the interaction between different WSLs with the help of a simple two-states model introduced earlier in Ref. [9] in relation to optical lattices. This model will allow us to distinguish between the cases of a weak, moderate, and strong interaction between the ladders. Going ahead, we note that the semiconductor SLs can show all three regimes of the interaction. This distinguishes them from the optical lattices, where the case of weak interaction is realized almost exclusively.

It is well known that the interaction between two resonances can be well modeled by a two-state system (see [15], for example). In this approach the problem reduces to the diagonalization of a $2 \times 2$ matrix, where the diagonal matrix elements correspond to the crossing (non-interacting) resonances. In our case, however, we have ladders of resonances. This fact can be properly taken into account by introducing the diagonal matrix in the form [9, 11]

$$
U_{0}=\exp \left(-i \frac{2 \pi H_{0}}{e d F}\right), \quad H_{0}=\left(\begin{array}{cc}
E_{1}^{*}-i \Gamma_{1} / 2 & 0 \\
0 & E_{2}^{*}-i \Gamma_{2} / 2
\end{array}\right) .
$$

It is easy to see that the eigenvalues $\lambda_{1,2}(F)=\exp \left[-i 2 \pi\left(E_{1,2}^{*}-i \Gamma_{1,2} / 2\right] / e d F\right)$ of $U_{0}$ correspond to the relative energies of WS-resonances and, thus, the matrix $U_{0}$ models two crossing ladders of resonances. (The resonance widths $\Gamma_{1,2}$ in Eq. (11) actually depend on $F$ in accordance with Eq. (5) but, considering a narrow interval of $F$, this dependence can be neglected.) Multiplying the matrix $U_{0}$ by the matrix

$$
U_{i n t}=\exp \left[i \epsilon\left(\begin{array}{ll}
0 & 1 \\
1 & 0
\end{array}\right)\right]=\left(\begin{array}{cc}
\cos \epsilon & i \sin \epsilon \\
i \sin \epsilon & \cos \epsilon
\end{array}\right)
$$

we introduce an interaction between the ladders.

The value $\epsilon=0$ obviously corresponds to non-interacting ladders. By choosing $\epsilon \neq 0$ but $\epsilon \ll \pi / 2$ we model the case of weakly interacting ladders. In this case the ladders show true crossing and the interaction affects only the stability of the ladders. Namely, in the limit $\epsilon \rightarrow 0$, the eigenvalues of the matrix $U_{0} U_{\text {int }}$ are

$$
\lambda_{ \pm}=\lambda_{1,2} \cos \epsilon\left(1+\frac{\tan ^{2} \epsilon}{1-\lambda_{1,2} / \lambda_{2,1}}\right) .
$$

It follows from Eq. (13) that at the points of crossing (where the phases of $\lambda_{1}$ and $\lambda_{2}$ coincide) the more stable ladder (let it be WSL with index 1, i.e. $\Gamma_{1}<\Gamma_{2}$ or $\left.\left|\lambda_{1}\right|>\left|\lambda_{2}\right|\right)$ is destabilized $\left(\left|\lambda_{+}\right|<\left|\lambda_{1}\right|\right)$ and, vice versa, the less stable ladder becomes more stable $\left(\left|\lambda_{-}\right|>\left|\lambda_{2}\right|\right)$. This situation is illustrated by the first row in Fig. 3, where we have a weak interaction between the ground and "first excited" WSLs. (Additional oscillations of $\Gamma_{2}$ are due to the interaction of first excited WSL with the second excited WSL, not shown in the figure.)

By increasing $\epsilon$ above $\epsilon_{c r}$,

$$
\tan ^{2} \epsilon_{c r}=\frac{\left(\left|\lambda_{1}\right|-\left|\lambda_{2}\right|\right)^{2}}{4\left|\lambda_{1} \lambda_{2}\right|}
$$


the case of moderate interaction, where the true crossing is substituted by an anticrossing, is met. As a consequence, the interacting WSLs exchange their stability index at the point of the avoided crossing. Let us also note that, according to Eq. (14), at fixed $\epsilon$ WS-resonances with comparable widths should show anticrossings while the resonances with essentially different widths should cross. Indeed, this is the case we typically observed in our numerical study of WS-resonances in the SL potential.

The maximal possible interaction is achieved by choosing $\epsilon=\pi / 2$. Then eigenvalues of the matrix $U_{0} U_{i n t}$ are $\lambda_{ \pm}= \pm i\left(\lambda_{1} \lambda_{2}\right)^{1 / 2}$ which corresponds to the "degenerate" resonances

$$
E_{ \pm}^{*}=\left(E_{1}^{*}+E_{2}^{*}\right) / 2 \pm e d F / 4, \quad \Gamma_{ \pm}=\left(\Gamma_{1}+\Gamma_{2}\right) / 2
$$

Relation (15) is illustrated by the second row in Fig. 3 where the strong interaction between the WSLs associated with the second and third Bloch bands produces a pair of locked WSLs, where the energy levels of one WSL are situated exactly in middle between the energy levels of the other WSL.

In conclusion, we note that in the general case, where one has more than two interacting WSLs, all three regimes of interaction (weak, moderate and strong) may present simultaneously. In particular, for the case depicted in the third row in Fig. 3 we have a strong interaction between the ladders originating from 2-nd and 3-rd and 4-th and 5-th Bloch bands, which build two locked [in the sense of Eq. (15)] pairs of ladders, a moderate interaction between these two pairs, and a weak interaction of the ground WSL with four above-barrier ladders.

\section{DENSITY OF STATES}

In this section we study the density of states of system (1). The analysis of the density of states is important because in the laboratory experiments one usually gains information about the density of states of the system rather than the resonances themselves.

Knowing the resonance energies $\mathcal{E}_{\alpha, l}=E_{\alpha}+e d F l-i \Gamma_{\alpha} / 2$, the fluctuating part of the density of states is given by

$$
\rho(E)=\frac{1}{2 \pi} \sum_{\alpha=1}^{\infty} \sum_{l=-\infty}^{\infty} \frac{\Gamma_{\alpha}}{\left(E_{\alpha}+e d F l-E\right)^{2}+\Gamma_{\alpha}^{2} / 4},
$$

where the sum over $\alpha$ is the sum over different WSLs. To avoid the convergence problem discussed in Sec. 3 we smooth the square-box potential (9) and substitute the unity step by the linear combination of hyperbolic tangents

$$
V(x)=\frac{V^{e}}{2}\left[\tanh \left(\frac{x+d / 4}{\sigma}\right)-\tanh \left(\frac{x-d / 4}{\sigma}\right)\right], \quad-d / 2<x<d / 2,
$$

where the parameter $\sigma$ defines the width of the transition region zone between the semiconductor layers. (For the used below $\sigma=2 d / \pi$ the shape of this potential is depicted in Fig. 1(b) by the dotted line.) The Fourier series of the function (17) has an effective cut-off above which the expansion coefficients decay exponentially. Then the upper limit in the sum over $\alpha$ in Eq. (16) can be set equal to this cut-off. For the sake of presentation it is also convenient to normalize the density of states $(16)$ to its mean value $\bar{\rho}=(e d F)^{-1}$.

Figure 4 shows the normalized electron density of states $\rho^{e}(E, F)$ as a gray-scaled map for strong fields, $F \geq 20$ $\mathrm{kV} / \mathrm{cm}$. It is seen in the figure, that $\rho^{e}(E, F)$ is dominated by the contributions from the under-barrier resonance $E_{1}^{*}$ and a pair of degenerate above-barrier resonances $E_{2}^{*}=E_{3}^{*}$. The effect of all other above-barrier resonances appears as weak ripples superimposed on these three fans. It is also interesting to note that the resonance $E_{1}^{*}$ can be easily followed up to the region of a very strong field $F>100 \mathrm{kV} / \mathrm{cm}$ where the potential wells of the combined potential $V(x)+e F x$ cannot support an under-barrier quasi-bound state in principle.

By a closer inspection of Fig. 4, a number of anticrossings between the "ground" and first two "excited" WSLs can be easily identified. These anticrossings are mainly reflected in a decrease of the contrast, which is a consequence of the increase of the resonance width $\Gamma_{1}$ occurring at the points of the avoided crossings (see inset of Fig. 4). It is also seen in the inset that the naively expected double-peak structure for the main resonance line may not appear in the energy dependence $\rho=\rho(E)$. In this sense, when the density of states is concerned, there are no big differences between anticrossings and actual crossings.

In conclusion of this section we would like to note that the results presented above cannot be considered as generic, because the structure of the above-barrier resonances is sensitive to the details of the SL potential. We have considered the case of a symmetric potential, where the width of the potential barriers coincides with the width of the potential wells. As an example, Fig. 5 shows the density of states for a asymmetric potential with the well to barrier ratio equal to $3.4 / 8$ (the lattice period $d$ is kept the same). In this case the presence of even harmonics in the series (17) affects 
the gaps at the center of the Brillouin zone, which removes the double degeneracy of the above-barrier resonances. It is seen in Fig. 5 that, unlike Fig. 4, the density of states is now dominated by the WSLs originating from the first $\left(E_{1}^{*} \approx \bar{E}_{1}=0.0284 \mathrm{eV}\right)$ and second $\left(E_{2}^{*} \approx \bar{E}_{2}=0.1214 \mathrm{eV}\right)$ Bloch bands.

\section{WAVE FUNCTIONS AND OVERLAP INTEGRAL}

We proceed with analysis of the wave function $\Psi_{\alpha, l}(x)$ of WS-states. Figure 6 shows $\left|\Psi_{1,0}(x)\right|^{2}$ for $F=5,10,25,100$ $\mathrm{kV} / \mathrm{cm}$. For $F<25 \mathrm{kV} / \mathrm{cm}$ these eigenfunctions resemble those of the tight-binding or single-band models [18, 19]. In particular, the phenomenon of Stark localization is clearly seen as $F$ is increased. The resonance origin of the depicted wave functions (i.e., the result beyond finite-band approximation) is reflected by the presence of the tail for $x<0$. This tail obviously corresponds to the probability tunneling out of the potential wells and its amplitude is directly related to the lifetime $\tau=\hbar / \Gamma$ of WS-states. When $F$ is increased, the amplitude of the probability flow gradually increases and eventually it becomes impossible to introduce a finite localization length of WS-states. This effect is an essence of the phenomenon which is often referred to as the delocalization of WS-states due to the Zener breakdown $[4,6]$. It is understood that the process of Stark localization is always accompanied by an increase of probability flow and, thus, a decrease of the lifetime of WS-state. For some system parameters (for example, smaller value of the potential barrier $V^{e}$ ) WS-states lose their stability before the Stark localization takes place. This is also the case for WS-states originating from the higher Bloch bands.

As far as the photo-current experiments with undoped SL is concerned, an important characteristic of SL is the overlap integral between the electron wave function $\Psi_{\alpha, l}^{e}(x)$ and the hole wave function $\Psi_{\alpha, l}^{h}(x)$. An analysis of this integral, which obviously depends only on the difference $L=l^{h}-l^{e}$ in the site indices of the wave functions, is our next aim. It should be noted that for the considered SL parameters $\left(d=114 \AA, a=b, V^{e}=0.0632 \mathrm{eV}, V^{h}=-0.0368 \mathrm{eV}\right.$, $m^{e}=0.067$, and $\left.m^{h}=-0.45\right)$ and considered interval of the static field $(F \geq 5 \mathrm{kV} / \mathrm{cm})$ the hole WS-states belonging to the ground hole WSL are essentially localized within one potential well. Thus the properties of the overlap integral discussed below are mainly defined by the localization of the electron WS-states.

As already mentioned in Sec. II, the right and left nonhermitian eigenfunctions of the Hamiltonian (1) generally do not coincide and, thus, the overlap integrals $\left\langle\Psi_{\alpha, l}^{h} \mid \Psi_{\beta, l+L}^{e}\right\rangle$ and $\left\langle\Psi_{\beta, l+L}^{e} \mid \Psi_{\alpha, l}^{h}\right\rangle$ may differ even in magnitude. (Here and below we use the Dirac notation for the right and left eigenfunctions.) A physical meaning, however, can only be attributed to the product of these integrals

$$
I_{\alpha, \beta}^{2}(L)=\left\langle\Psi_{\alpha, l}^{h} \mid \Psi_{\beta, l+L}^{e}\right\rangle\left\langle\Psi_{\beta, l+L}^{e} \mid \Psi_{\alpha, l}^{h}\right\rangle .
$$

The absolute values of $I_{\alpha, \beta}^{2}(L)$, which can be interpreted as the squared transition matrix elements, define the intensities of the absorption lines. In the low field limit, the integral (18) can be calculated on the basis of the tight-binding model, because in this limit both the right and left resonance eigenfunctions approach the stationary states of the single-band model. For a high field, however, it has to be calculated numerically.

Figure 7 shows the absolute values of the transition matrix elements $I_{\alpha, \beta}(L)=\sqrt{I_{\alpha, \beta}^{2}(L)}$ for $\alpha=\beta=1$ as a function of $L$. The chosen values of static field corresponds to the regimes of low $(F=5 \mathrm{kV} / \mathrm{cm})$, medium $(F=10,25$ $\mathrm{kV} / \mathrm{cm})$, and high $(F=100 \mathrm{kV} / \mathrm{cm})$ fields. It is seen in the figure that in the low field regime many transitions are involved. The total number of the transitions is obviously given by the localization length of electron WS-states, which can be well estimated by using the tight-binding model. When the static field is increased ( $F=10$ and 25 $\mathrm{kV} / \mathrm{cm}$ ) the localization length of WS-states decreases and the vertical transition $L=0$ becomes dominant. However, in addition to the vertical transition a long tail of tilted transition for $L>0$ appears $(F=25$ and $100 \mathrm{kV} / \mathrm{cm})$. This phenomenon is obviously related to a field-induced delocalization of WS-states illustrated in Fig. 6.

To conclude this section we shall briefly discuss the phase of the overlap integral

$$
I_{\alpha, \beta}^{2}(L)=\left|I_{\alpha, \beta}^{2}(L)\right| \exp \left[i \phi_{\alpha, \beta}(L)\right]
$$

When the transition to an unstable resonance is involved, the phases $\phi_{\alpha, \beta}(L)$ generally differ from zero. Because for large values of $F$ all WS-resonances are actually the unstable above-barrier resonances, this fact should be properly taken into account when the high field regime is considered (see next section). Beside this, the effect of phases was found to be extremely important at the point of anticrossings. In fact, at the points of anticrossings the distance between resonances is typically smaller then the widths of the resonances involved and, therefore, an interference may occur. We mainly met the case of destructive interference, i.e. the phases of the corresponding matrix elements tend to be opposite to each other. 


\section{WANNIER-STARK FAN}

In this section we use the preceding results to calculate the absorption spectra for the system (1). According to Refs. $[4,7]$ the absorption spectra of the semiconductor superlattice is defined by the one-dimensional absorption

$$
D(\omega)=\pi \iint \mathrm{d} E^{e} \mathrm{~d} E^{h}\left|\int \mathrm{d} x \Psi^{h *}\left(x ; E^{h}\right) \Psi^{e}\left(x ; E^{e}\right)\right|^{2} \rho^{e}\left(E^{e}\right) \rho^{h}\left(E^{h}\right) \delta\left(E^{e}-E^{h}+E_{g}-\hbar \omega\right) .
$$

In this equation, which is just Fermi's golden rule, $\Psi(x ; E)$ are the hermitian eigenfunctions of the Hamiltonian $(1)$ ( $E$ is real and continuous), $\rho^{e, h}(E)$ are the electron and hole densities of states, $\omega$ is the frequency of radiation, and $E_{g}$ stands for the energy gap in the bulk semiconductor. Although Eq. (20) has a simple form, the numerical evaluation of the double integral in this equation appears to be a rather difficult problem [4, 20].

Here we employ a different method of calculating the one-dimensional absorption spectrum, which is based on the notion of quantum resonances. In fact, since the density of states (16) has simple poles at $\mathcal{E}=E_{\alpha}+e d F l-i \Gamma_{\alpha} / 2$, the integral (20) can be estimated by using the residuum theorem. Presenting the $\delta$-function in Eq. (20) in the form

$$
\delta\left(E^{e}-E^{h}+E_{g}-\hbar \omega\right)=\lim _{\epsilon \rightarrow 0} \frac{1}{\pi} \operatorname{Im}\left(\frac{1}{E^{e}-\left(E^{h}\right)^{*}+E_{g}-\hbar \omega-i \epsilon}\right)
$$

(the formal operation of complex conjugation for $E^{h}$ ensures the correct limit on $\epsilon$ when the energies are complex), we obtain

$$
D(\omega) \sim \sum_{\alpha, \beta} \sum_{L} \operatorname{Im}\left[\frac{I_{\alpha, \beta}^{2}(L)}{\left(E_{\beta}^{e}-E_{\alpha}^{h}+e d F L+E_{g}-\hbar \omega\right)-i\left(\Gamma_{\beta}^{e}+\Gamma_{\alpha}^{h}\right) / 2}\right],
$$

where the coefficients $I_{\alpha, \beta}^{2}(L)$ are given by Eq. (18). The proportionality sign, which formally appears because we drop the sum over $l$ in the route from Eq. (20) to Eq. (22), denotes that the absorption $D(\omega)$ is proportional to the size (length) of the system. We would like to note that Eq. (22) adopts for the case of the semiconductor superlattice an analytical expression derived earlier for the decay spectra in the optical superlattices [10]. In Ref. [10] this equation was obtained by using the nonhermitian perturbation theory. Here we based our derivation on Fermi's golden rule (20) which is, however, also limited to low intensities of the driving field. Thus, the present approach and the one in Ref. [10] are complementary to each other.

Equation (22) has a simple physical meaning. In fact, by neglecting the phases of the overlap integrals, it reduces to

$$
D(\omega) \sim \sum_{\alpha, \beta} \sum_{L}\left|I_{\alpha, \beta}^{2}(L)\right| \frac{\Gamma_{\alpha}^{h}+\Gamma_{\beta}^{e}}{\left(E_{\beta}^{e}-E_{\alpha}^{h}+e d F L+E_{g}-\hbar \omega\right)^{2}+\left(\Gamma_{\alpha}^{h}+\Gamma_{\beta}^{e}\right)^{2} / 4}
$$

Then the absorption spectra is a sum of the Lorentzians associated with vertical $(L=0)$ and tilted $(L \neq 0)$ transitions between $\alpha$-th hole and $\beta$-th electron WS-states. Being included, the phases of the overlap integrals typically cause non-Lorentzian shapes of the absorption lines - a phenomenon referred to as Fano resonances in the semiconductor superlattices [21]. Beside this, as mentioned above, they take into account the destructive interference occurring at the points of avoided crossings.

As an example, Fig.8 shows the one-dimensional absorption $D(\omega, F)$ calculated on the basis of Eq. (22) for SL with parameters of Fig. 7. Because in the high field regime the ground electron and hole wave functions are essentially localized within one well, $L=0$ transitions between the ground electron and hole states dominate in the absorption spectrum. The weak feathers above and below the main feather obviously correspond to $L= \pm 1$ and $L= \pm 2$ transition between the same WSLs. (Also a sign of another fan, associated with $\alpha=2, \beta=1$ transitions, is seen.) It is interesting to note that, although there are no absorption lines associated with $\beta>1$ electron WSLs in Fig. 8, the existence of these above-barrier WSLs is confirmed indirectly by the complicated structure of the visible absorption lines. In particular, comparing Fig. 8 with Fig. 4, one can easily identify the anticrossings of under-barrier (for $F \rightarrow 0$ ) WS-resonances $E_{1}^{*}$ with two above-barrier WS-resonances $E_{2}^{*}=E_{3}^{*}$. These anticrossings originate a characteristic "broken feather" structure of the $L=0$ absorption line, recently detected in the experiment [7].

\section{CONCLUSION}

Let us summarize the results obtained. 
We calculated the resonance WS-states in a semiconductor SL and studied the dependence of the resonance width (inverse lifetime of WS-states) on the amplitude of the static field. When the static field is varied, the resonance width is found to show multiscale oscillations, which are superimposed on the smooth overall behavior predicted by Landau-Zener theory. We explain these multiscale oscillations by the interaction between different WSLs which is studied in some detail by using a simple matrix model.

Although the general structure of the electron or hole density of states is obvious (a periodic sequence of peaks associated with WS-resonances) there were no general methods to calculate it [22]. Section 5, where we analyzed the dependence of the density of states on both the energy and amplitude of the static field, fills this gap in the theory.

Next we studied the overlap integral between the electron and hole resonance WS-states. In particular, it was shown that in the high field regime the transitions "up the ladder" are enhanced in comparison with the transitions "down the ladder". This asymmetry is a consequence of the field-induced delocalization of WS-states and has to be distinguished from the asymmetry caused by the electron-hole Coulomb interaction [19]. We also stressed an importance of complex phases of the overlap integral which are responsible for interference effects, occurring at (anti)crossings of different WSLs, and non-Lorentzian shape of the absorption lines.

A method of calculating the shape of absorption line is suggested. Using this method one can easily construct the Wannier-Stark fan for an arbitrary wide range of the static field. Previously this was done only by a numerical evaluation of the Fermi golden rule [4]. However, the Fermi golden rule approach gives little insight into the physics of Wannier resonances. Beside this, the numerical method of Ref. [4] is essentially a finite-size-box quantization method, where the results should be controlled against the variation of the box size. In contrast, the method described in Sec. 7 relies on the resonance WS-states, which assumes an infinite size of the system from the very beginning.

A final remark concerns the Coulomb interaction, which was neglected throughout the paper. Because we were mainly interested in the regime of high static field where the Coulomb binding energy is smaller than the electrostatic energy $\Delta E=e d F$, this assumption is partially justified. Nevertheless, it would be interesting to study the effect of the Coulomb interaction on the lifetime of the exciton WS-states. This problem is reserved for future.

\section{REFERENCES}

[*] Also at L. V. Kirensky Institute of Physics, 660036 Krasnoyarsk, Russia. e-mail: kolovsky@physik.uni-kl.de

[1] G. H. Wannier, Phys. Rev. 117, 432 (1960).

[2] E. E. Mendez, F. Agullo-Rueda, and J. M. Hong, Phys. Rev. Lett. 60, 2426 (1988).

[3] J. Leo and A. MacKinnon, J. Phys.: Condens. Matter 1, 1449 (1989).

[4] S. Glutsch and F. Bechstedt, Phys. Rev. B 60, 16584 (1999).

[5] A. Sibille, J. F. Palmier, and F. Laruellle, Phys. Rev. Lett. 80, 4506 (1998).

[6] R. Rosam, F. Löser, V. G. Lyssenko, K. Leo, S. Glutsch, F. Bechstedt, and K. Köhler, Phys. Stat. Sol. (b) 221, $463(2000)$.

[7] R. Rosam, D. Meinhold, F. Löser, V. G. Lyssenko, S. Glutsch, F. Bechstedt, F. Rossi, K. Köhler, and K. Leo, Phys. Rev. Lett. 86, 1307 (2001).

[8] M. Glück, A. R. Kolovsky, H. J. Korsch, and N. Moiseyev, Eur. Phys. J. D 4, 239 (1998).

[9] M. Glück, A. R. Kolovsky, and H. J. Korsch, Phys. Rev. Lett. 82, 1534 (1999).

[10] Glück, M. Hankel, A. R. Kolovsky, and H. J. Korsch, Journal of Optics B: Quantum and Semiclassical Optics 2, 612 (2000).

[11] Glück, A. R. Kolovsky, and H. J. Korsch, Journal of Optics B: Quantum and Semiclassical Optics 2, 694 (2000).

[12] General properties of the quantum resonances are discussed, for example, in N. Moiseyev, Physics Reports 302, 211 (1998).

[13] C. Zener, Proc. R. Soc. London Ser. A 145, 523 (1934).

[14] The advantage of the evolution operator over the Hamiltonian is that it recovers the translational symmetry of the problem. In the tight-binding approximation the properties of the evolution operator $U\left(T_{B}\right)$ are discussed in Qian Niu, Phys. Rev. B 40, 3625 (1989).

[15] M. Wagner and H. Mizuta, Phys. Rev. B 48, 14393 (1993).

[16] At the first order perturbation theory the values of the energy gaps are given by the values of the Fourier expansion coefficients of a periodic potential [N. W. Ashcroft, N. D. Mermin,Solid State Physics, (1972)].

[17] Within the tight-binding approach the interaction between WSLs was studied in G. Bastard, R. Ferreira, S. Chelles, and P. Voisin, Phys. Rev. B 50, 4445 (1994).

[18] J. Bleuse, G. Bastard, and P. Voisin, Phys. Rev. Lett. 60, 220 (1988).

[19] M. M. Dignam and J. E. Sipe, Phys. Rev. B 43, 4097 (1991).

[20] S. Glutsch and D. S. Chemla, Phys. Rev. B 54, 11592 (1996). 
[21] C. P. Holfeld, F. Löser, M. Sudzius, K. Leo, D. M. Whittaker, and K. Köhler, Phys. Rev. Lett. 81, 874 (1998).

[22] We note, however, the paper M. C. Chang and Q. Niu, Phys. Rev. B 48, 2215 (1993), where the density of states was calculated for the periodic potential consisting of a chain of $\delta$-function barriers. 

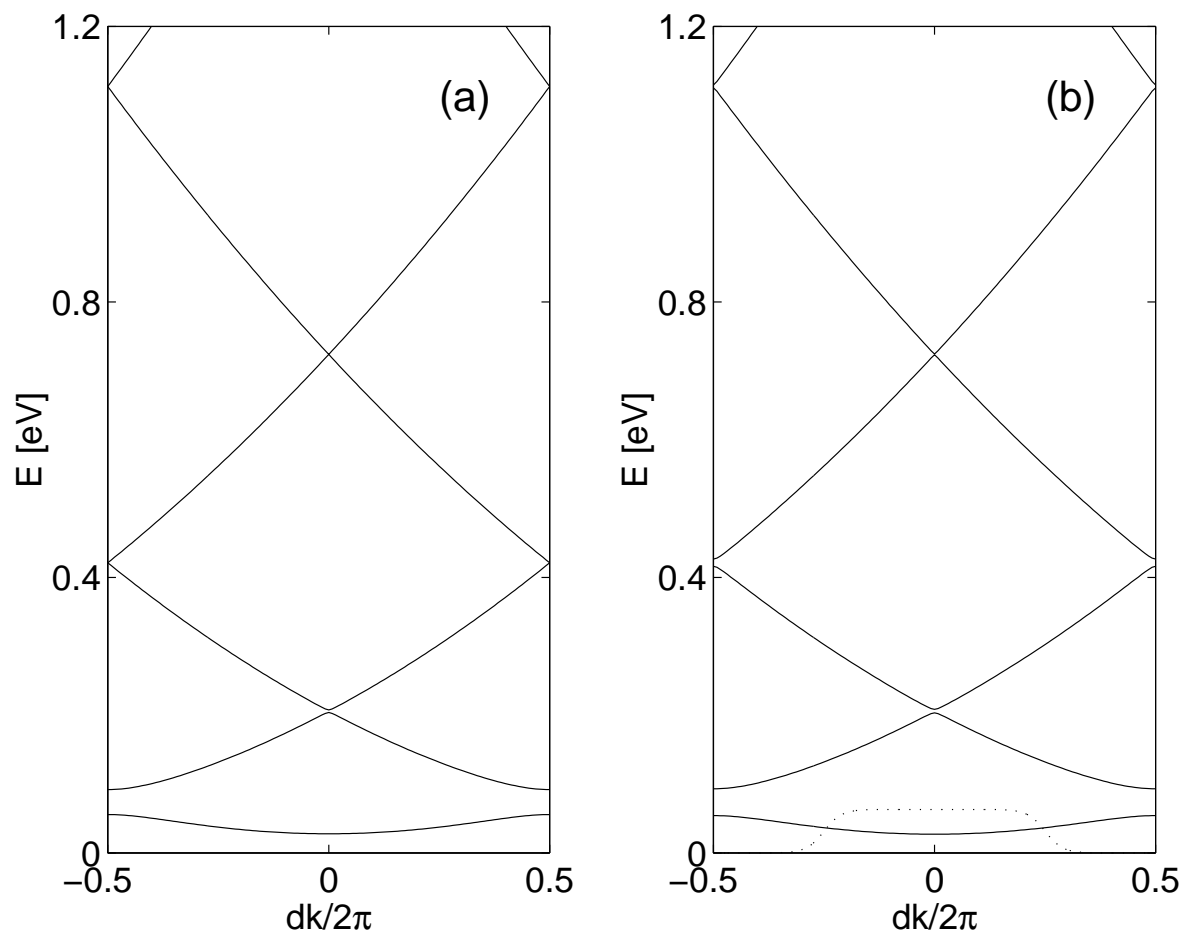

FIG. 1: Bloch band structure of the electron in the cosine potential $V(x)=V^{e}[0.5+(2 / \pi) \cos (2 \pi x / d)]$ (a) and square-box potential (9) with equal thickness of the layers (b). The parameters are $V^{e}=0.0632 \mathrm{eV}, d=114 \AA$ and $m^{e}=0.067$ of the electron mass. Additionally, the dotted line in the right panel shows the smoothed square-box potential used later on. 

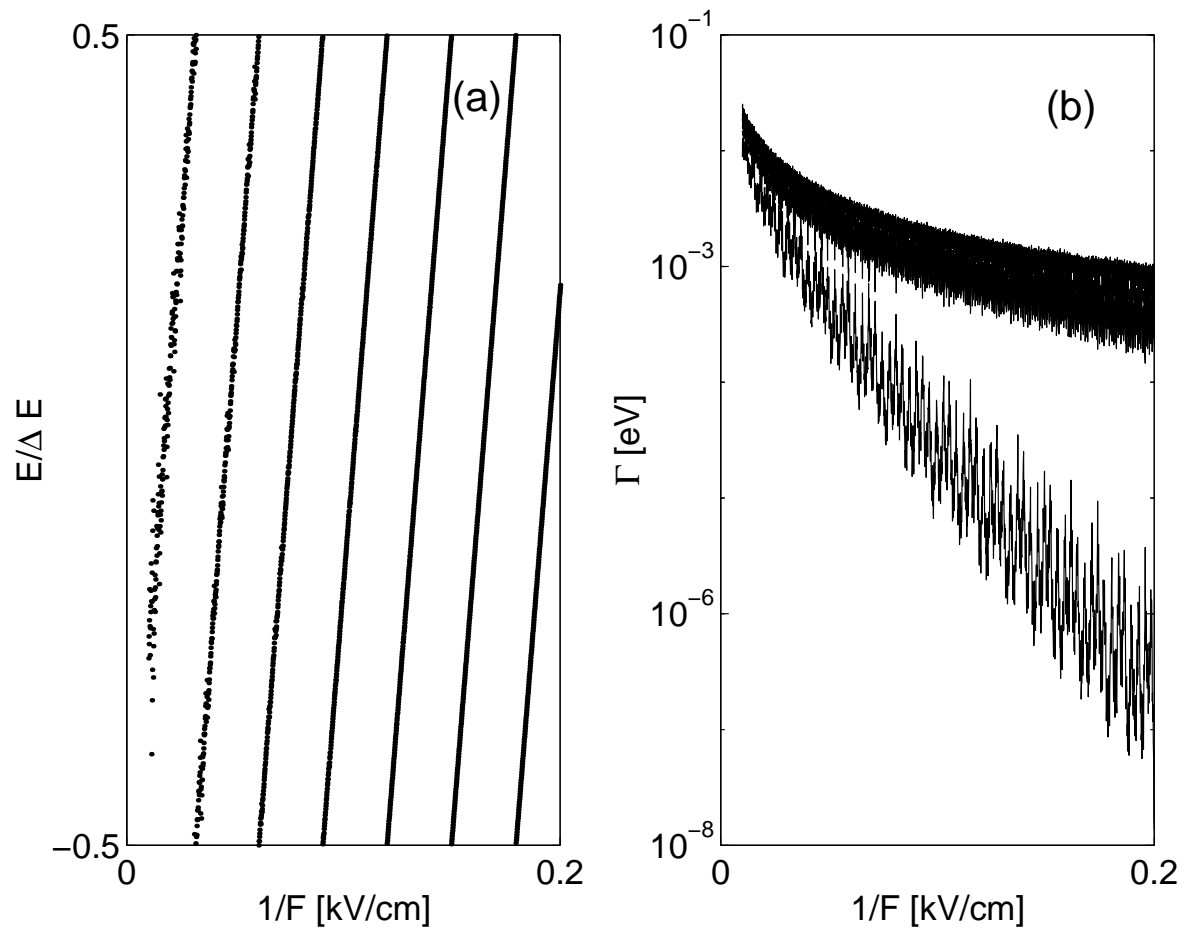

FIG. 2: (a) Level position of the most stable electron WSL reduced to the fundamental energy interval $-\Delta E / 2 \leq$ $E<\Delta E / 2, \Delta E=e d F$. (b) The widths of the first seven most stable WS-resonances as a function of $1 / F$. 

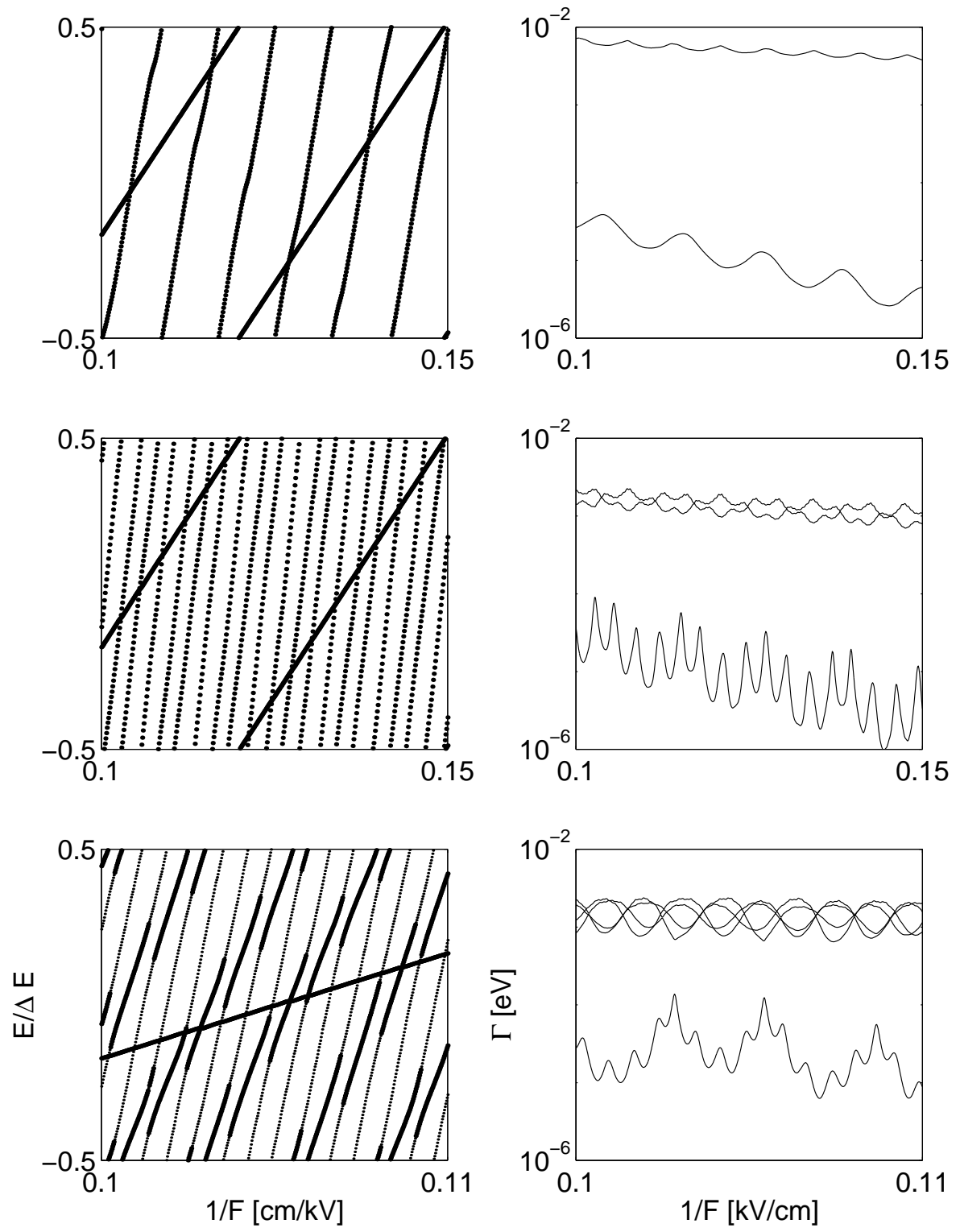

FIG. 3: A fragment of the dependence of $E_{\alpha}$ and $\Gamma_{\alpha}$ on $1 / F$ calculated for $M=1$ (upper row), $M=3$ (middle row), and $M=5$ (lower row), where $M$ is the upper limit in the sum (10). In the low raw two less stable resonances are marked by a smaller dots. 


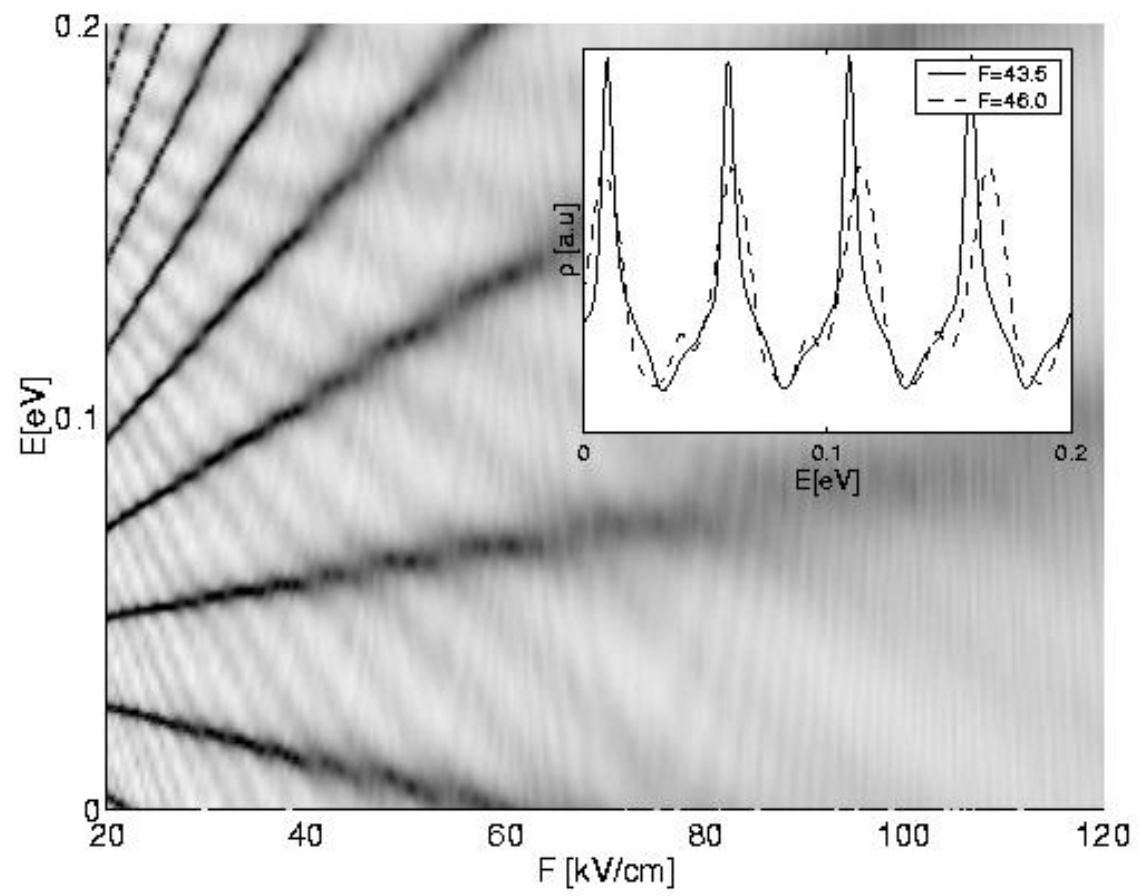

FIG. 4: Grey-scaled map of the electron density of states (16) for the smoothed square-box potential (17) with $\sigma=2 d / \pi$. The inset shows $\rho(E)$ for $F$ around (dashed line) and far (solid line) from the anticrossing at $F \approx 46$ $\mathrm{kV} / \mathrm{cm}$.

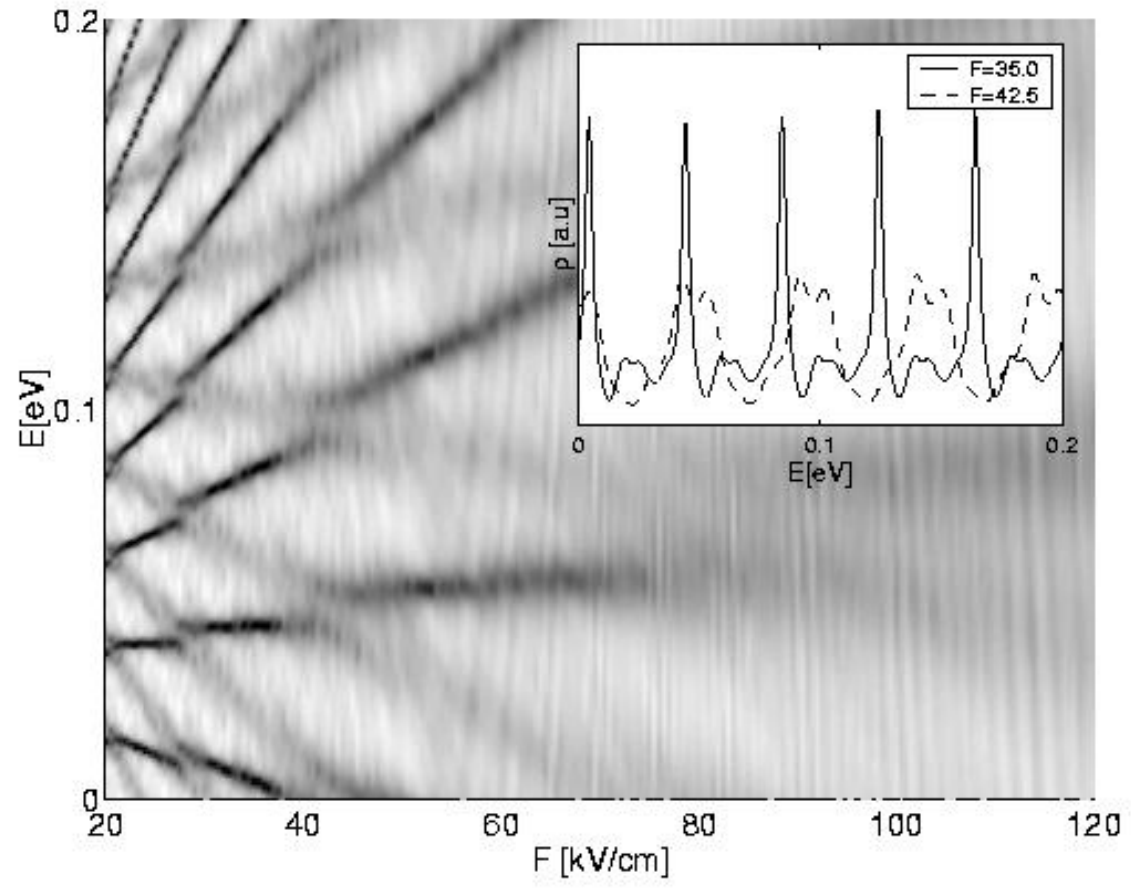

FIG. 5: Same as Fig. 4, but for an asymmetric potential with $a / b=3.4 / 8$. 


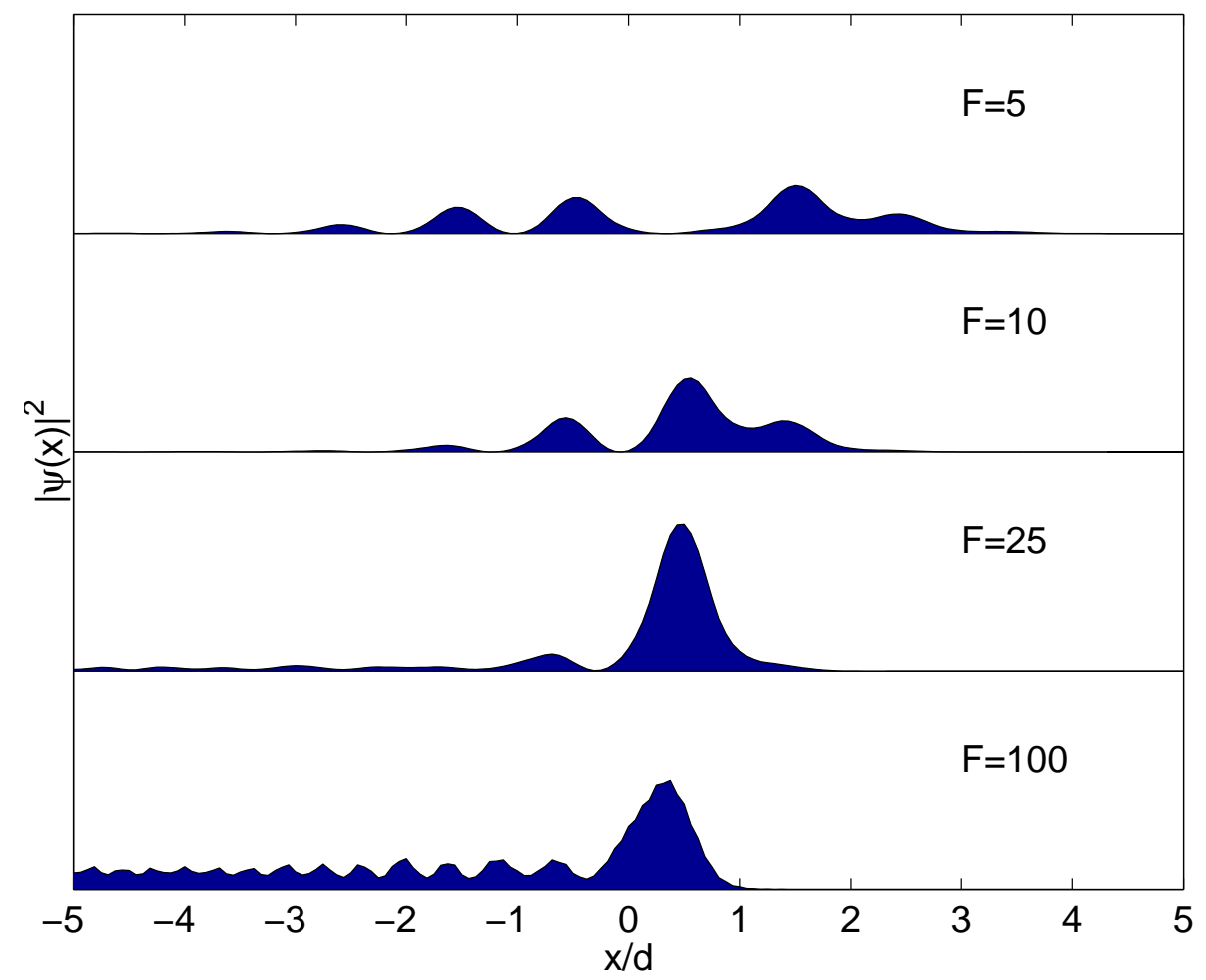

FIG. 6: Wave functions of ground electron WS-state for different values of the static field $F$.
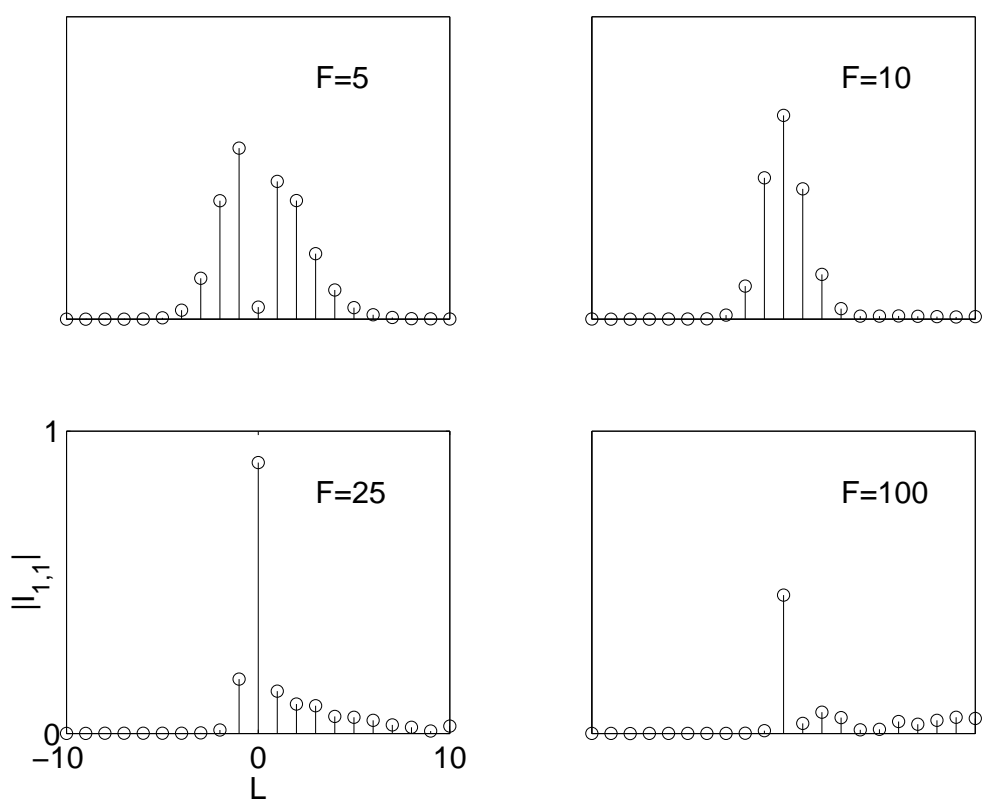

FIG. 7: Square root of the absolute value of the overlap integral (18) for $\alpha=\beta=1$ as a function of $L$ for four different values of the static field $F=5,10,25,100 \mathrm{kV} / \mathrm{cm}$. SL parameters are $d=114 \AA, a=b, V^{e}=0.0632 \mathrm{eV}$, $V^{h}=-0.0368 \mathrm{eV}$, and $m^{e}=0.067, m^{h}=-0.45$ of the electron mass. 


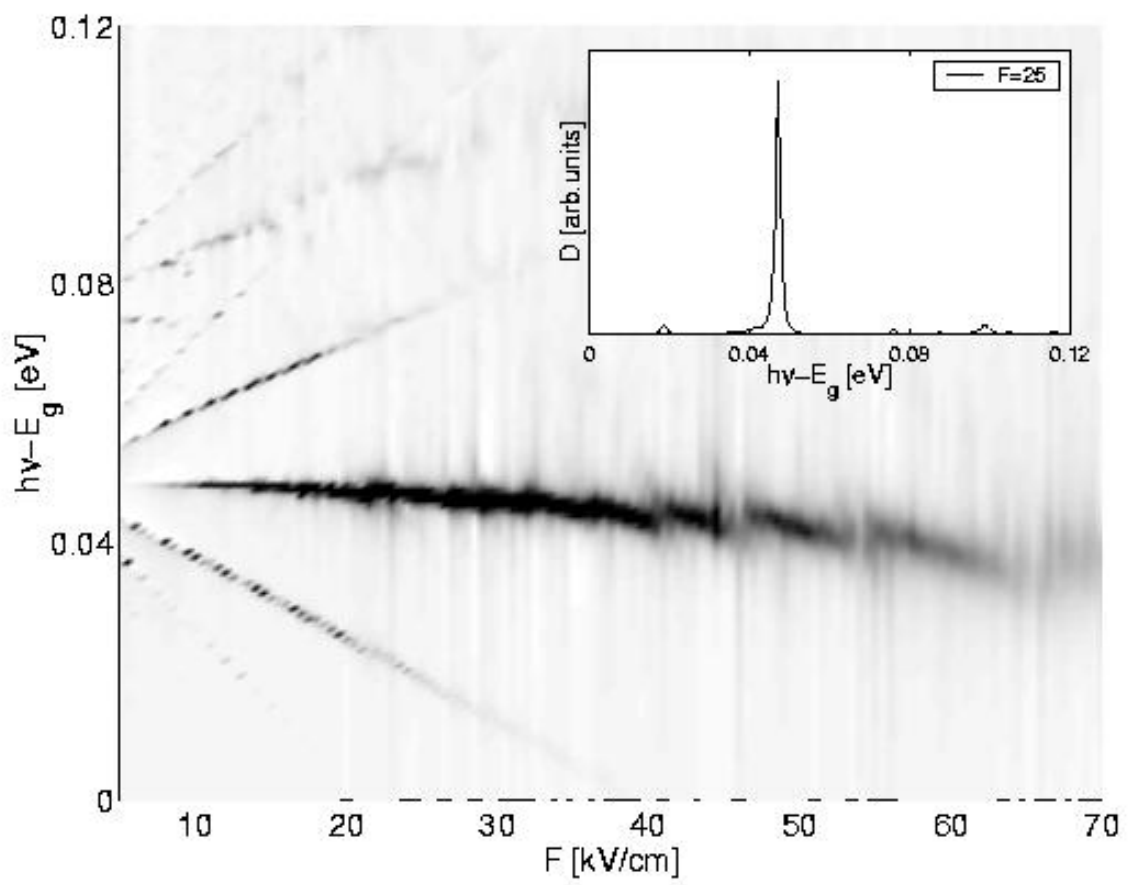

FIG. 8: Grey-scaled map of the one-dimensional absorption spectra as a function of the static field $F$ and photon energy $h \nu$. The inset shows the shape of absorption lines for $F=25 \mathrm{kV} / \mathrm{cm}$. 